\title{
The Bicuspid Aortic Valve Syndrome: A Multifaceted Challenge: Do
}

\section{We Know Enough?}

\author{
Petropoulos AC* \\ The "Aziz Aliyev" national postgraduate medical and CME training center, Baku, \\ Azerbaijan
}

*Corresponding author: Ass. Prof./Dr. Andreas C Petropoulos, Senior Consultant Pediatrician, Fetal, Pediatric and Congenital Cardiologist, Merkezi klinika, XMSK hospital, The "Aziz Aliyev" national postgraduate medical and CME training center, Baku, Azerbaijan, Tel: +994502869828; Email: andrepetropoulos@gmail.com

Abbrevations: BAov: Bicuspid Aortic valve; CHD: Congenital Heart Disease; HLHS: Hypoplastic Left Heart Syndrome; PDA: Patent Ductus Arteriosus; VSD: ventricular septal defect; CoA: Coarctation of the Aorta; CVS: cardiovascular system; AAo: Ascending Aorta; AVR: Aortic Valve Replacement; TAVR: Transcatheter Aortic Valve Replacement.

\section{Editorial}

Among the most interesting heart diseases, one can be described as the most fascinating of all. The Bicuspid Aortic valve (BAov) earns easily this distinguish. This can be explained in many ways. As the commonest of all Congenital Heart Disease (CHD). Presenting in any agefrom fetal to late adulthood, even...never! With a variety of features as severe as Hypoplastic Left Heart Syndrome (HLHS) to undetected valvular disease or subclinical endocarditis, mentioning a few of its many presentations. Taking in consideration that the adverse cardiovascular outcomes in patients suffering from it, are more common than previously thought, therefore due to its high prevalence it presents a large burden on cardiovascular care.

Although, Leonardo da Vinci first described, and Sir William Osler pronounced it: the most common CHD, we still have more questions than answers regarding its nature.

BAov has an estimated worldwide prevalence of $1-2 \%$ of the general population and $3 / 1$ preference in males.
The exact pathogenesis of the formation of it is not yet fully understood. Possible a genetic component exists, given its association with others CHD's such as Patent Ductus Arteriosus (PDA), ventricular septal defect (VSD) and coarctation of the aorta $(\mathrm{CoA})$.

Up to date, genetic causes and clinical implications for the majority of BAov patients remain largely unknown. Evidence of an autosomal dominant pattern of BAov inheritance with variable expression and incomplete penetrance in families has been described. Additional, mutations in NOTCH1 - a mechanosensory receptor found in arteries - has been found to associate with the presence of a BAov and valvular calcium-deposition reactivation. Some rare family cases, specific mutations or distinction in GATA5 - a transcription factor that regulates the proper embryological development of the cardiovascular system (CVS) - have been associated with BAov and aortopathy.

We believe that a multifactorial event during the embryogenesis of the semilunar valves of the CVS, leads to a formation of a fusion between the aortic cusps, creating the defect.

The 2008 classification by Schaefer, et al. describes four sub-types: I. Fusion seen between right - left cusps (70- 80\% cases), II. Fusion seen between right - noncoronary cusps $(20-29 \%$ cases), III. $1 \%$ of cases with fusion between left - noncoronary cusps. IV. Sub-type without any raphe; often referred to as a true BAov, in comparison to the term "functional BAov" describing subtypes I-III, were 3 cusps can be seen with the valve closed 


\section{Open Access Journal of Cardiology}

but only two functions when opening. True BAov have only two symmetrical leaflets and two sinuses of Valsalva. Clinically, sub-type I is more likely to suffer stenosis in adults while subtype II valves will present pathology in younger ages.

Another intriguing issue is that in many cases of a BAov, histological abnormalities that lead to a combined aortopathy are found. This presents with dilatation of the Ascending Aorta (AAo). Although the most common area to dilate is the tubular AAo, the entire AAo may be affected, including the sinuses of Valsalva and sinotubular junction. Finally, a subgroup of BAov patients who exhibit dilatation of the sinuses of Valsalva preferentially exists. This pattern is common in sub-type I BAov and male gender.

These pathological anatomical alternations are thought not only to be due to the altered flow in the AAo, but also due to cellular structural abnormalities including decreased fibrillin, causing smooth muscle cell detachment, and cell death.

The existence of the two "functional" or true cusps, even in early stages have normal function or minimally dysfunction - seen by echocardiography - exhibit asymmetrical systolic excursion with marked bending strain in systole, high stress in the area around the ralph of the conjoined cusp and uneven systolic flow patterns. All these and maybe more unknown to us elements lead over time to an intrinsic stenosis and or regurgitation of the valve with or with not aortic route dilatation.

The above mentioned genetic factors in combination to embryological mostly unknown elements accumulate to a conundrum of the true nature of this disease. It is possible that the BAov may be a by-product of a more widespread genetic alteration involving the aorta and other structures of the developing heart. Alternatively, maybe, they are more restricted factors limited to the valve, that lead to distinct types of primary BAov disease, mostly stenosis.

Compering pathophysiology elements we can distinct three disease forms. Firstly, a primary valvular type, presents from birth or gradually, as aging increases its amount of stenosis. In this form the most probable in future the need for aortic valve replacement (AVR) is anticipated. Studies have shown this happening in 25$65 \%$ of patients in a 25 year period.

The second form is a mixed valvular-aortopathy that predominates to BAov regurgitation (BAovR+) and AAo aneurysm formations with augmented aortic dissection risk in future. Studies have proven that $15-30 \%$ will need surgical repair of the BAovR+ between the 4th - 5th decade of their life. $45 \%$ of the group will suffer aneurysm formations while $26 \%$ will need to be operated during that same age period.

A third more complex form exists, often diagnosed by its symptomatic presentation in childhood. This form can explain the combination of CHD and BAov, using its best example, Shone's complex (series of at least 4 left heart, co-existing structure stenosis).

From the above mentioned we seem to have two major clinical phenotypes. A "pediatric form", presenting earlier in life. A large study from autopsy specimens, calculated a prevalence of $6.7 \%$ of these complex CHD including a BAov as one of their elements. Examples are: BAov with: CoA (51.5\%), with VSD (20.5\%), with CoA and VSD, with PDA. BAov has been also reported as a part of transposition of the great arteries (1\%), HLHS, complete atrioventricular canal defect, Ebstein's anomaly, partial or total anomalous pulmonary venous drainage, tetralogy of Fallot, double-outlet right ventricle, left ventricular septal diverticulum. It can also be a part of genetic syndromes that involve CHD. Such are Turners (30\%), William's, Down's, Marfan's and Loeys-Dietz.

These patients also present with a higher incidence of left dominance coronary artery pattern (57\%) with higher incidence of immediate bifurcation of the left main coronary artery, and higher incidence of mean length of the left main coronary significantly shorter to $10 \mathrm{~mm}$. Anomalous origins of both right and left coronary arteries, anomalous origins of the left circumflex artery and single left coronary artery, have been reported. Spontaneous coronary artery dissection may occur also.

An "adult form", in which: isolated stenosis (36\%), regurgitation (44\%), both (20\%), worsening by age at least and possible by accumulation of cardiovascular risk factors that accelerate atherosclerosis. Additional to these more "silent" clinical presentations, formation of aneurysms (17-45\%), rapture of them (10\%), endocarditis (0.3-2\%/yearly risk) and thrombotic events have been reported.

BAov natural history has been evaluated in several studies. It is known to be variable and dependent on associated abnormalities, age of patient and anatomical subtype. It can range from severe valval stenosis in childhood to asymptomatic minimal disease in patient's in 


\section{Open Access Journal of Cardiology}

their 70's. Many of the adult patients (approximately $75 \%$ ) have progressive fibrocalcific valval stenosis and aortopathy, eventually requiring surgical treatment. This usually leads to presentation in middle age; in contrast only $2 \%$ of children have isolated significant BAov disease.

Diagnosis is based on clinical suspicion by medical, family history or incidentally finding during a well-baby or physical examination. Late presenters are common, mostly with "isolated- adult" valval form. The mainstay of diagnosis is echocardiography (Transthoracic or Transesophageal) which can provide diagnosis in most patients detecting also progression of aortopathy, before complications occur. Recently, the use of metalloproteinase plasma assays, computer tomography and magnetic resonance imaging, have been introduced in clinical practice diagnosis and risk stratification.

Decisive treatment is only by surgical means. Medicines as: b-blockers, ACE-I, ARDS and Statins, have been used to alleviate symptoms and slow progression.

The 2014 ACC/AHA and the 2017 ESC guidelines for the management of patients with valvular heart disease address all the indications, types of surgery and/or intervention strategies in adult patients.

In children valve replacement is not encouraged as physical development, outgrows the prosthetic valve. Strategies to repair the stenotic or regurgitant valve or even the use of a sub-coronary Ross procedure - by an experienced surgeon- with pulmonary autograft, should be used because of the excellent postoperative long-term results. Due to the lack of valve calcification in children, balloon valvuloplasty is possible and can be in same cases the strategy of choice. Studies have shown good follow-up in both the immediate and medium terms, follow-up. This can buy time bridging the patient before needing surgery.

In summary, there are still large gaps in understanding the pathophysiology of BAov-associated valvopathy aortopathy. Today, finding of a BAov in any age patient, must be approached as a Syndrome and not a defined disease. Most recent studies have frequently produced conflicting findings regarding the subsequent development of BAov disease. Neither the prevalence, appearance, nor relation to valve morphology has been defined and accepted with certainty. The relative contribution of genetic factors and hemodynamic stresses to the aortopathy of BAov remains unclear in many details.

Finally, there is a trend towards Transcatheter aortic valve replacement (TAVR) and classical surgeries for BAov -associated valvopathy and aortopathy respectively. Newer therapies will need to be compared against the "traditional" Bentall surgery, and likely hybrid procedures such as Bentall surgery with bioprosthetic aortic valve replacement and subsequent follow-up of TAVR procedure.

The only robust data on the subject of the syndrome of the BAov is that we still have many to learn in how to deal efficiently with a condition in which its high prevalence, many subtypes and presentations in any age group highlights it as a major public health treat.

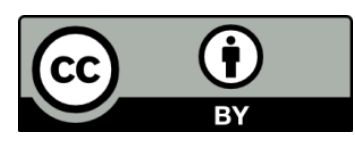

